\title{
Fano-type interference in quantum dots coupled between metallic and superconducting leads
}

\author{
J. Barański and T. Domański \\ Institute of Physics, M. Curie-Sktodowska University, 20-031 Lublin, Poland
}

(Dated: October 5, 2018)

\begin{abstract}
We analyze the quantum interference effects appearing in the charge current through the double quantum dots coupled in $T$-shape configuration to an isotropic superconductor and metallic lead. Owing to proximity effect the quantum dots inherit a pairing which has the profound influence on nonequilibrium charge transport, especially in the subgap regime $|V|<\Delta /|e|$. We discuss under what conditions the Fano-type lineshapes might appear in such Andreev conductance and consider a possible interplay with the strong correlation effects.
\end{abstract}

\section{INTRODUCTION}

Heterostructures with nanoobjects (such as quantum dots, nanowires, molecules, etc) hybridized to one conducting and another superconducting electrode seem to be promising testing fields where the strong electron correlations (responsible e.g. for Coulomb blockade and Kondo physics [1]) can be confronted with the superconducting order [2]. Coulomb repulsion between electrons in the solid state physics is known to suppress the local ( $s$-wave) pairing and, through the spin exchange mechanism, eventually promotes the intersite ( $d$-wave) superconductivity [3]. Mutual relation between such repulsion and the local pairing is however rather difficult for studying, both on theoretical grounds and experimentally. In nanoscopic heterostructures some of these limitations can be overcome by a suitable adjustment of the hybridization and the gate-voltage positioning of energy levels involved in the charge transfer [4]. They enable a controllable changeover between the Kondo regime and opposite case dominated by the induced on-dot pairing.

Quantum dot (QD) coupled with the strength $\Gamma_{N}$ to metallic conductor $(\mathrm{N})$ and with $\Gamma_{S}$ to superconducting electrode $(\mathrm{S})$ can exhibit the features characteristic both for the on-dot pairing and the Kondo effect (including their coexistence) [5]. Their efficiency depends on the ratio $\Gamma_{S} / \Gamma_{N}$. In the limit $\Gamma_{S} \gg \Gamma_{N}$ the underlying physics is controlled by on-dot pairing and manifests itself e.g. by the particle-hole splitting of the quasiparticle levels. On the other hand for $\Gamma_{S} \ll \Gamma_{N}$ the strong correlations take over. Non-trivial aspects related to such interplay between the Coulomb interactions and the proximity induced on-dot pairing has been addressed theoretically using various methods like: the mean field slave boson approach [6], the noncrossing approximation [7], perturbative scheme [2, 8], constrained slave boson technique 9], numerical renormalization group [10 12] and other [1316]. Also the cotunneling regime of a Coulomb blockaded quantum dot sandwiched between a normal and superconducting lead, where charge fluctuations are strongly suppressed, has been discussed emphasizing the role of in-gap resonances [17].

As far as the experimental situation is concerned it has been less intensively explored. The earliest transport

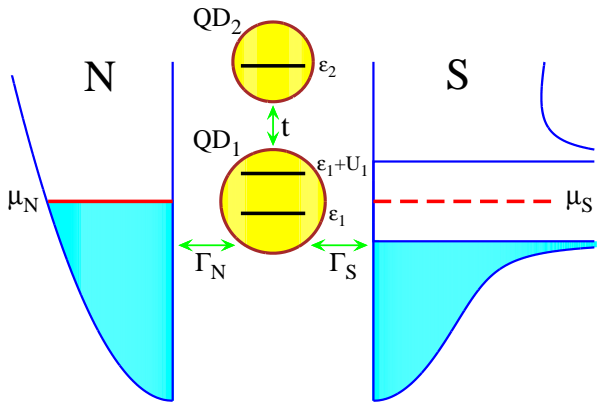

FIG. 1: (color online) Scheme of the double quantum dot in $T$-shape configuration coupled to the conducting $(\mathrm{N})$ and superconducting (S) leads, where interference effects originate from the interdot hopping $t$.

measurements for N-QD-S interface have been obtained using the multi-wall carbon nanotube deposited between $\mathrm{Au}$ and $\mathrm{Al}$ electrodes [18]. Those investigations concentrated however on the specific regime $k_{B} T_{K} \geq \Delta$, when the Coulomb correlations dominated over the proximity effect. Other studies of the same group have been done for similar structures replacing a metallic electrode by a ferromagnet [19]. Several recent efforts focused on the multiterminal structures involving two normal and one superconducting electrodes as useful schemes for realization of: the crossed Andreev reflections tunable via gate voltages [20], the Cooper pair splitters [21], and the QD spin valves [22].

Very useful understanding of a subtle interplay between the correlations and the induced on-dot pairing has been gained from recent measurements by R.S. Deacon et al [5]. The authors have explored the subgap transport originating from the Andreev-type scattering processes for several representative ratios $\Gamma_{S} / \Gamma_{N}$ using the self-assembled InAs quantum dots deposited between the golden $(\mathrm{N})$ and aluminium $(\mathrm{S})$ electrodes. Their measurements provided the unambiguous experimental evidence for: a) particle-hole splitting of the subgap conductance of the Andreev states when $\Gamma_{S} \geq \Gamma_{N}$, and b) enhancement of the zero-bias Andreev conductance due to formation of the Kondo resonance at the Fermi level of metallic 
lead, as has been qualitatively suggested by our studies [16] and also indicated by other groups [23].

The present work extends our former studies by taking into account interference effects arising from the additional degrees of freedom. As the simplest prototype for Fano-type interference [24] we consider the setup (see figure 1) with a side-attached quantum dot contributing an extra pathway for electrons transmitted between the metallic and superconducting leads. Our analysis is complementary to the previous study by Y. Tanaka et al who considered the double quantum dots coupled between $\mathrm{N}$ and $\mathrm{S}$ leads in a $T$-shape setup but assuming $U_{1}=0$, $U_{2} \neq 0$ [25] and in a series configuration [26].

In section 2 we introduce the microscopic model and briefly outline basic notes on the nonequilibrium subgap transport. In the next section 3 we discuss a unique way in which the Fano-type lineshapes might be observed in Andreev conductance, focusing on the uncorrelated quantum dots. In the last part (section 4) we discuss the influence of correlations at the interfacial quantum dot which seem to have remarkable influence on the low bias transport. We end with the summary and suggestions for the future studies.

\section{THE MODEL}

For description of the heterojunction illustrated in figure 1 we use the Hamiltonian

$$
\hat{H}=\hat{H}_{N}+\hat{H}_{N-D Q D}+\hat{H}_{D Q D}+\hat{H}_{S-D Q D}+\hat{H}_{S}
$$

where the double quantum dot (DQD) is represented by

$$
\hat{H}_{D Q D}=\sum_{\sigma i} \epsilon_{i} \hat{d}_{i \sigma}^{\dagger} \hat{d}_{i \sigma}+U_{1} \hat{n}_{1 \uparrow} \hat{n}_{1 \downarrow}+\left(t \hat{d}_{1 \sigma}^{\dagger} \hat{d}_{2 \sigma}+\text { h.c. }\right) \text {. }
$$

The energies of each $(i=1,2)$ quantum dot electrons are denoted by $\varepsilon_{i}$ and $t$ stands for the usual interdot hopping. We restrict considerations of the correlation effects (section 4) to the Coulomb repulsion $U_{1}$ between opposite spin electrons $\sigma=\uparrow, \downarrow$ at the interfacial quantum dot.

The external reservoirs $\mathrm{N}$ and $\mathrm{S}$ of charge carriers are described by $\hat{H}_{N}=\sum_{\mathbf{k}, \sigma} \xi_{\mathbf{k} N} \hat{c}_{\mathbf{k} \sigma N}^{\dagger} \hat{c}_{\mathbf{k} \sigma N}$ and correspondingly $\hat{H}_{S}=\sum_{\mathbf{k}, \sigma} \xi_{\mathbf{k} S} \hat{c}_{\mathbf{k} \sigma S}^{\dagger} \hat{c}_{\mathbf{k} \sigma S}-\sum_{\mathbf{k}} \Delta \hat{c}_{\mathbf{k} \uparrow S}^{\dagger} \hat{c}_{-\mathbf{k} \downarrow S}^{\dagger}+$ $\Delta^{*} \hat{c}_{-\mathbf{k} \downarrow S} \hat{c}_{\mathbf{k} \uparrow S}$ assuming the isotropic energy gap $\Delta$. As usually $\xi_{\mathbf{k} \beta}=\varepsilon_{\mathbf{k} \beta}-\mu_{\beta}$ denote the electron energies measured from the individual chemical potentials $\mu_{\beta}$ which become detuned $\mu_{N}-\mu_{S}=e V$ if a bias $V$ is applied across the junction inducing the nonequilibrium charge flow $I(V)$. Fano-type quantum interference effects originating from the hopping $t$ to side-coupled quantum dot $i=2$ are discussed here assuming that only the interfacial quantum dot $i=1$ is directly coupled to external leads

$$
\hat{H}_{\beta-D Q D}=\sum_{\mathbf{k}, \sigma}\left(V_{\mathbf{k} \beta} \hat{d}_{1 \sigma}^{\dagger} \hat{c}_{\mathbf{k} \sigma \beta}+\text { h.c. }\right) \text {. }
$$
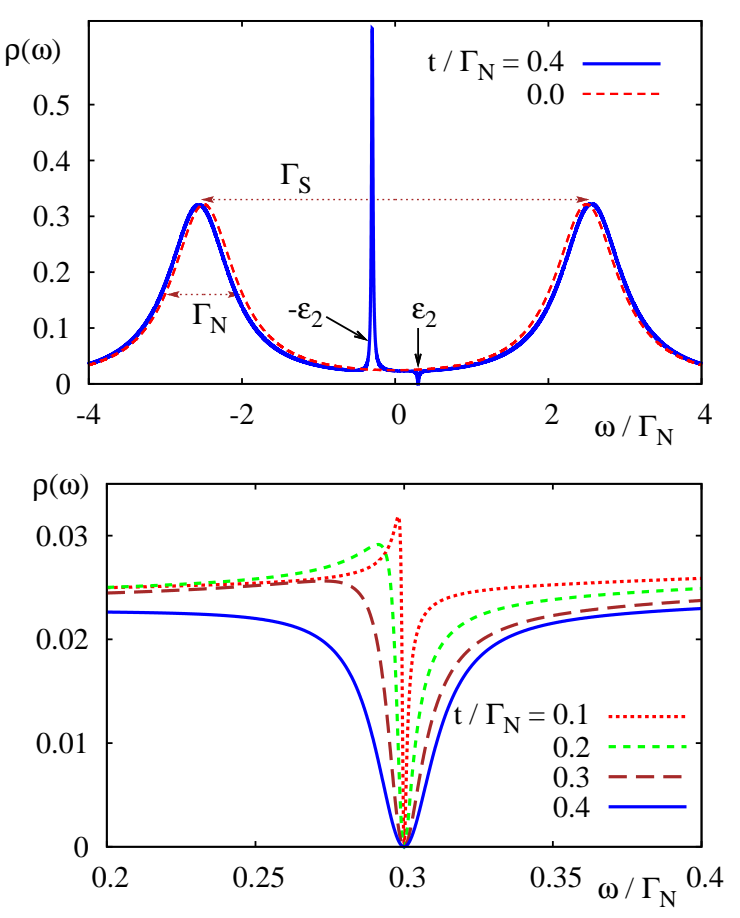

FIG. 2: (color online) Density of states $\rho(\omega)$ of the interfacial $i=1 \mathrm{QD}$ obtained in equilibrium conditions for $\varepsilon_{1}=0, \varepsilon_{2}=$ $0.3 \Gamma_{N}, \Gamma_{S}=5 \Gamma_{N}$ and a few values of the interdot hopping $t$.

In the wide-band limit approximation it is convenient to introduce the structureless coupling constants $\Gamma_{\beta}=$ $2 \pi \sum\left|V_{\mathbf{k} \beta}\right|^{2} \delta\left(\omega-\xi_{\mathbf{k}}\right)$ which shall be used here as the energy units.

Interplay between the proximity induced on-dot pairing, the correlations and the quantum interference effects can be in practice detected by measuring the differential conductance $d I(V) / d V$. Particularly valuable for this purpose is the low voltage (subgap) regime $|e V| \ll \Delta$. Under such conditions the charge current is provided by the anomalous Andreev scattering in which electrons from the metallic lead are converted into the Cooper pairs in superconductor with a simultaneous reflection of the electron holes back to the normal lead. On a formal level the resulting Andreev current can be expressed by the Landauer-type formula 9, 13]

$$
I_{A}(V)=\frac{2 e}{h} \int d \omega T_{A}(\omega)[f(\omega-e V, T)-f(\omega+e V, T)],
$$

where $f(\omega, T)$ is the Fermi distribution function and the transmittance $T_{A}(\omega)=\Gamma_{N}^{2}\left|G_{12}(\omega)\right|^{2}$ depends on the offdiagonal part (in the Nambu notation) of the retarded Green's function (5) of the interfacial quantum dot.

\section{FANO RESONANCES}

Fano resonances appear in many physical systems due to the quantum interference of the waves transmitted res- 


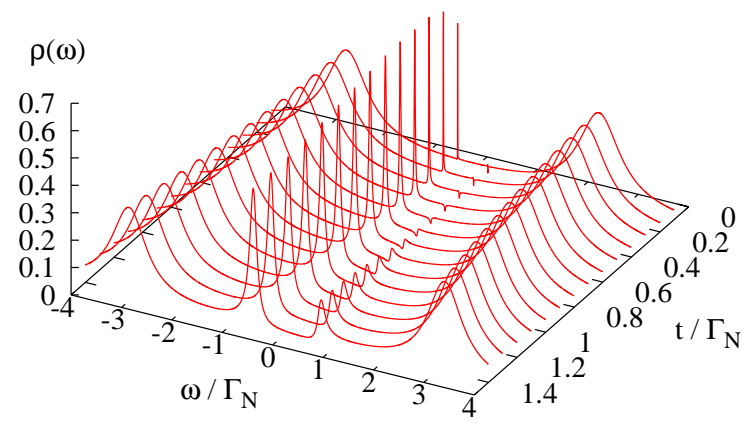

FIG. 3: (color online) Changeover of the interfacial quantum dot spectrum from the Fano (resonance and antiresonance) lineshapes to the effective four-peak structure upon increasing the interdot hopping $t$ for the same parameters as in figure 2

onantly via some discrete energy level combined with transmittance contributed from a continuum of other states. In nanoscale physics such resonances are feasible in a variety of constructions [24]. Fano lineshapes are present for instance in the electron transport when two external electrodes are in parallel coupled through a quantum dot and directly via a shortcut bridge [27]. Another simple possibility takes place in the electron tunneling using two quantum dots with considerably different linebroadenings [28, 29]. In the latter case the narrower level is responsible for forming the Fano resonance on a background of the broader level.

In this work we want to analyze similar interference effects appearing in the anomalous Andreev current, which is very specific because of the particle and hole degrees of freedom mixed with one and other. To have a clear picture of the underlying physics let us start by considering the noninteracting case $U_{i}=0$ when the Green's functions of each quantum dot can be determined exactly.

Electron transport of the setup shown in figure 1 is determined by effective properties of the interfacial quantum dot. For this purpose we compute the matrix Green's function $\boldsymbol{G}\left(\tau_{1}, \tau_{2}\right)=-\hat{T}_{\tau}\left\langle\hat{\Psi}\left(\tau_{1}\right) \hat{\Psi}^{\dagger}\left(\tau_{2}\right)\right\rangle$ introducing the standard spinor notation $\hat{\Psi}^{\dagger}=\left(\hat{d}_{1 \uparrow}^{\dagger}, \hat{d}_{1 \downarrow}\right)$ and $\hat{\Psi}=\left(\hat{\Psi}^{\dagger}\right)^{\dagger}$. In the equilibrium conditions $\mu_{N}=\mu_{S}$ this function depends only on the time difference and its Fourier transform obeys the following Dyson equation

$$
\boldsymbol{G}(\omega)^{-1}=\left(\begin{array}{cc}
\omega-\varepsilon_{1} & 0 \\
0 & \omega+\varepsilon_{1}
\end{array}\right)-\boldsymbol{\Sigma}^{0}(\omega)-\boldsymbol{\Sigma}^{U}(\omega)
$$

where the term $\boldsymbol{\Sigma}^{0}(\omega)$ corresponds to the selfenergy of noninteracting case $\left(U_{1}=0\right)$ and $\boldsymbol{\Sigma}^{U}(\omega)$ accounts for the correlation effects (discussed in section IV). Focusing on the deep subgap regime $|\omega| \ll \Delta$ we obtain that $\boldsymbol{\Sigma}^{0}(\omega)$

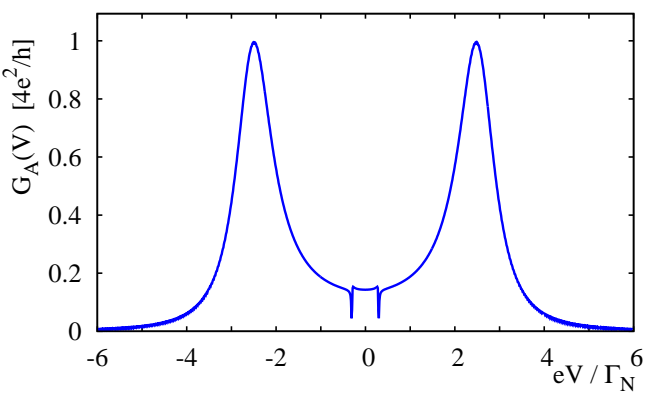

FIG. 4: (color online) The differential Andreev conductance $G_{A}(V)$ versus the bias $V$ revealing the quasiparicle peaks (near $\pm \sqrt{\varepsilon_{1}^{2}+\left(\Gamma_{S} / 2\right)^{2}}$ ) and Fano-type lineshapes (near $\pm \varepsilon_{2}$ ) for the set of parameters used in figure 2 and $t=0.1 \Gamma_{N}$.

simplifies to (see the appendix)

$$
\Sigma^{0}(\omega)=\left(\begin{array}{cc}
-\frac{i \Gamma_{N}}{2}+\frac{t^{2}}{\omega-\varepsilon_{2}} & -\frac{\Gamma_{S}}{2} \\
-\frac{\Gamma_{S}}{2} & -\frac{i \Gamma_{N}}{2}+\frac{t^{2}}{\omega+\varepsilon_{2}}
\end{array}\right) .
$$

When the interference effects caused by the hopping $t$ to the side-coupled QD are neglected the expression (6) becomes static ( $\omega$-independent) and nontrivial physics of this, so called atomic superconducting limit, has been explored in detail by several groups [2, 10, 30] including ourselves [16].

Taking into account the quantum interference $t \neq 0$ we show in figure 2 the proximity induced on-dot pairing [formally arising from the off-diagonal parts of [6] ] illustrating the energy spectrum $\rho(\omega)=-\frac{1}{\pi} \operatorname{Im} \boldsymbol{G}_{11}\left(\omega+i 0^{+}\right)$ obtained for strong coupling to the superconducting lead $\Gamma_{S}=5 \Gamma_{N}$. Such coupling $\Gamma_{S}$ is responsible for the particle-hole splitting of the effective quasiparticle states formed at $\pm \sqrt{\varepsilon_{1}^{2}+\left(\Gamma_{S} / 2\right)^{2}}$ whereas the coupling $\Gamma_{N}$ controls their broadening. In the particular case $\varepsilon_{1}=0$ the quasiparticle peaks appearing at $\pm E_{1}$ (where $\left.E_{1} \equiv \sqrt{\varepsilon_{1}^{2}+\Gamma_{S}^{2} / 4}\right)$ are symmetric, but for arbitrary $\varepsilon_{1}$ they are weighted by the corresponding BCS coefficients $u^{2}, v^{2}=\frac{1}{2}\left(1 \pm \varepsilon_{1} / E_{1}\right)$ [16]. On top of such behavior we clearly notice that hopping to the side-coupled quantum dot induces additional features appearing in the effective spectrum near $\pm \varepsilon_{2}$ as the Fano resonance and antiresonance. For the case of both metallic leads there would survive just the single Fano structure at $\varepsilon_{2}$ which in very pedagogical way has been discussed by R. Žitko 28].

Fano-type lineshapes (see the lower panel in figure 21) are present only in the weak hopping regime $t \ll \Gamma_{N}$. For increasing $t$ the Fano structures gradually evolve into separate quasiparticle peaks illustrated in figure 3. Physically this can be assigned to the induced pairing on the side-attached QD $\left\langle\hat{d}_{2 \downarrow} \hat{d}_{2 \uparrow}\right\rangle \neq 0$ transmitted there indirectly via the interfacial quantum dot. Such effect again qualitatively differs from the structures of the DQD coupled to both metallic leads [28, 29].

Interrelation between the interference and proximity effect can be practically investigated by measuring the 


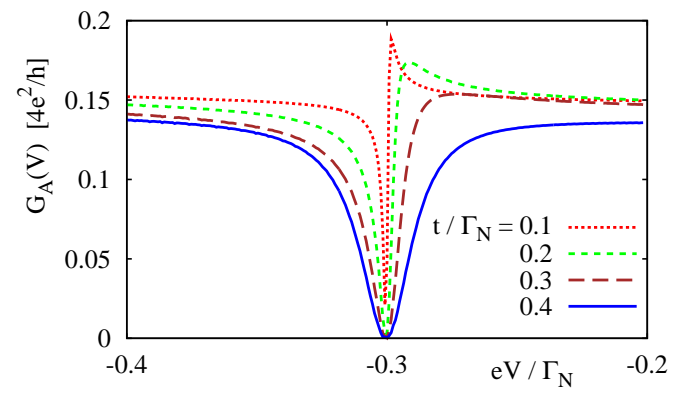

FIG. 5: (color online) Differential conductance $G_{A}(V)$ of the subgap Andreev current versus the source-drain bias $V$ in a vicinity of the Fano structure appearing at $V= \pm \varepsilon_{2} / e$.

tunneling current. In figure 4 we show bias voltage $V$ dependence of the differential Andreev conductance $G_{A}(V)=d I_{A}(V) / d V$ determined at zero temperature from (4) over a broad regime covering both the subgap quasiparticle peaks. Figure 5 illustrates the resulting Fano-type lineshapes $G_{A}=G_{0} \frac{(x+q)^{2}}{x^{2}+1}+G_{1}$ nearby $-\varepsilon_{2}$, where $x=\left|e V+\varepsilon_{2}\right| / \Gamma_{N}$ and the asymmetry parameter $q$ gradually decreases upon increasing the hopping integral $t$. Our results can be thought as extension of the predictions obtained for the normal electron tunneling using the $T$-shape DQD coupled to both metallic leads [28, 32] onto the anomalous Andreev current where the particle hole mixing has the essential importance.

\section{INTERPLAY WITH CORRELATIONS}

Coulomb repulsion between electrons of opposite spins can have an important influence on the spectral and transport properties of various nanostructures. For the case of quantum dots coupled to both conducting leads such interactions are known to be responsible for: a) the charging effect (if a given energy level $\varepsilon_{i}$ is attempted to be occupied by more than a single electron this costs the system an extra energy $\left.U_{i}\right)$, b) the Kondo effect when the singlet state is formed between $\mathrm{QD}$ and itinerant electrons from the leads [31]. In spectroscopic properties they are manifested by appearance of the Coulomb satellite around $\omega=\varepsilon_{i}+U_{i}$ and the narrow Kondo resonance at the Fermi level. For heterostructures with the superconducting electrodes the situation is more complex due to a competition between the induced on-dot pairing and Coulomb repulsion.

The rich interplay between the quantum interference, correlations and proximity effect for the configuration shown in figure 1 have been so far addressed using the density functional technique [33] (which does not capture the Kondo physics) and by the numerical renormalization group calculations [25]. In latter case the authors focused on $U_{1}=0, U_{2} \neq 0$ when the side-attached quantum dot can indirectly form the Kondo state with electrons of the metallic lead affecting the Andreev transport.
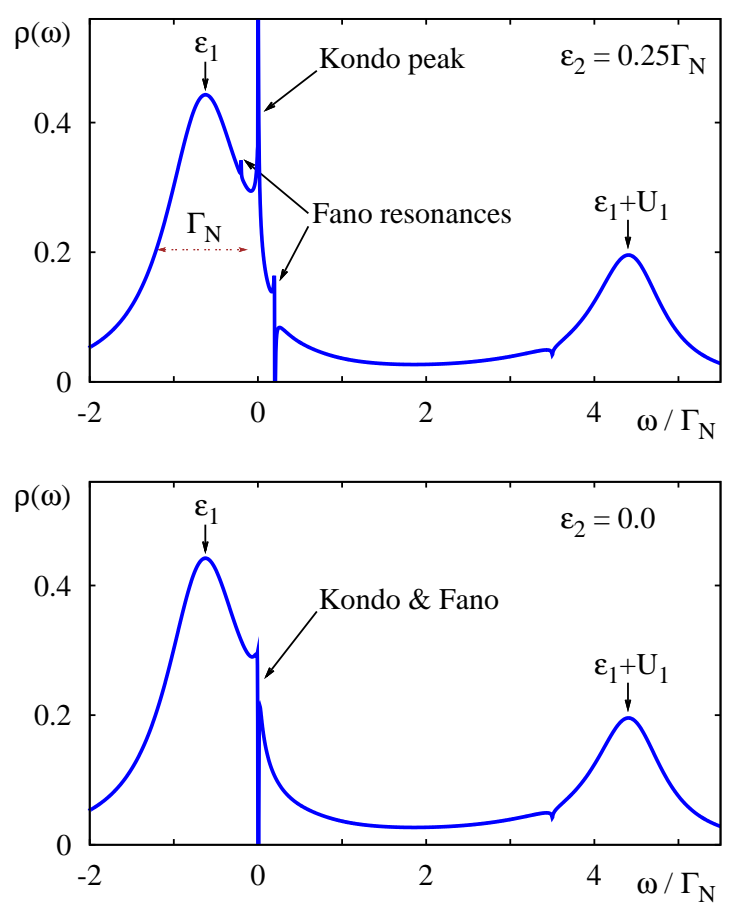

FIG. 6: (color online) Density of states $\rho(\omega)$ of the correlated interfacial $\mathrm{QD}$ in the Kondo regime obtained for: $\varepsilon_{1}=-0.75 \Gamma_{N}, \Gamma_{S}=\Gamma_{N}, U=5 \Gamma_{N}, t=0.1 \Gamma_{N}$ and temperature $k_{B} T=0.001 \Gamma_{N}$. The upper panel shows the spectrum for $\varepsilon_{2}=0.25 \Gamma_{N}$ with two Fano structures appearing at $\pm \varepsilon_{2}$ aside the Kondo peak. The lower plot corresponds to $\varepsilon_{2}=0$ when both the Kondo and Fano structures are superimposed.

To account for the correlation effects predominantly originating from the interfacial quantum dot we extend here the procedure previously used by us for studying the single quantum dot [16]. The main idea is to approximate the correlation selfenergy $\boldsymbol{\Sigma}^{U}(\omega)$ by the diagonal matrix

$$
\Sigma^{U}(\omega) \simeq\left(\begin{array}{cc}
\Sigma_{N}(\omega) & 0 \\
0 & -\Sigma_{N}^{*}(-\omega)
\end{array}\right)
$$

Such assumption (applied also in the NRG studies [10]) can be thought as the simplest ansatz for the many-body selfenergy $\boldsymbol{\Sigma}^{U}(\omega)$ allowing to combine the proximity effect (6) with the correlations, brought separately from the particle and hole channels. In more advanced treatments one should take into account the possible feedback effects between these normal and anomalous channels. We nevertheless hope that by imposing (7) we can get some insight at least on a qualitative level which might stimulate the future studies.

Within qualitative studies of the correlation effects we can describe the Coulomb blockade and Kondo effects using the following equation of motion expression [31]

$\Sigma_{N}(\omega)=\omega-\varepsilon_{1}-\frac{\left[\tilde{\omega}-\varepsilon_{1}\right]\left[\tilde{\omega}-\varepsilon_{1}-U_{1}-\Sigma_{3}(\omega)\right]+U_{1} \Sigma_{1}(\omega)}{\tilde{\omega}-\varepsilon_{1}-\left[\Sigma_{3}(\omega)+U_{1}\left(1-n_{1, \sigma}\right)\right]}(8)$

where $\Sigma_{\nu=1,3}(\omega)=\sum_{\mathbf{k}}\left|V_{\mathbf{k} N}\right|^{2}[f(\omega, T)]^{\frac{3-\nu}{2}}\left[\left(\omega-\xi_{\mathbf{k} N}\right)^{-1}+\right.$ 


\section{SUMMARY}
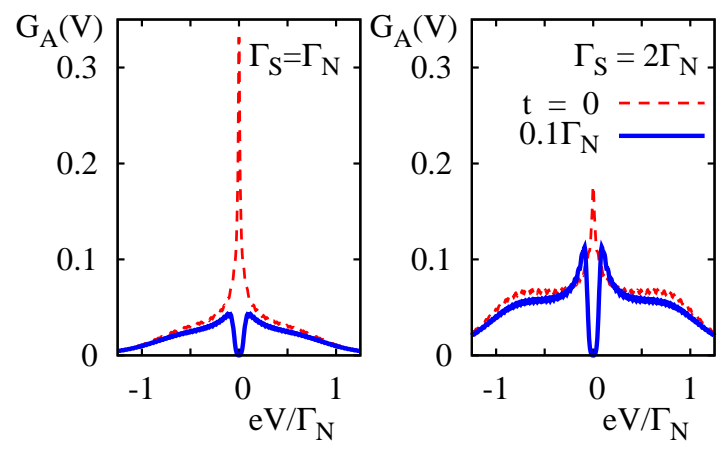

FIG. 7: (color online) Andreev conductance $G_{A}(V)$ expressed in units of $4 e^{2} / h$ obtained for $\Gamma_{S}=\Gamma_{N}$ (left h.s. panel) and $\Gamma_{S}=2 \Gamma_{N}$ (panel on the right). The thin dashed lines correspond to $t=0$ whereas the thick solid lines show influence of the Fano-type interference for $t=0.1 \Gamma_{N}$. In both plots we used $\varepsilon_{2}=0$ and the same model parameters as in figure [6

$\left.\left(\omega-U_{1}-2 \varepsilon_{1}+\xi_{\mathbf{k} N}\right)^{-1}\right], n_{1, \sigma}=\left\langle\hat{d}_{1 \sigma}^{\dagger} \hat{d}_{1 \sigma}\right\rangle$ and $\tilde{\omega}=\omega+\frac{i \Gamma_{N}}{2}$. We explored the interfacial quantum dot spectrum and the related transport properties at $k_{B} T=0.001 \Gamma_{N}$, i.e. well below the Kondo temperature. Specific numerical computations have been done for $\varepsilon_{1}=-0.75 \Gamma_{N}, U_{1}=5 \Gamma_{N}$ and symmetric coupling to both external leads giving the optimal conditions for any low-bias features in the Andreev current [5, 16]. This ratio $\Gamma_{S} / \Gamma_{N} \sim 1$ is a reason why the particle-hole splitting is hardly visible, but otherwise (for larger $\Gamma_{S}$ ) the Kondo peak is either reduced or completely absent [16].

Upper panel in the figure 6 illustrates the Fano resonance/antiresonance around $\pm \varepsilon_{2}$ (where $\left.\varepsilon_{2}=0.25 \Gamma_{N}\right)$ obtained for the hopping $t=0.1 \Gamma_{N}$. These Fano-type interference objects appear on top of the characteristic spectrum consisting of the Kondo resonance and the broad quasiparticle peaks seen at $\varepsilon_{1}$ and its Coulomb satellite at $\varepsilon_{1}+U_{1}$. Such spectrum is the result of interference effects discussed in the preceding section and the correlation features. The lower panel of figure[6]shows the very specific situation $\varepsilon_{2}=0$ when the Kondo and Fano structures coincide with each other. The Fano-type resonance seems to play then a dominant role.

Differential conductance of the Andreev current (4) for the $T$-shaped double quantum dot system (11) is shown in figure 7 . We notice a clear suppression of the zero-bias peak (present for the single quantum dot case $t=0$ as indicated by the dashed lines) because of a destructive influence of the Fano-type interference (the solid lines). The subgap Andreev current is thus very sensitive to the interplay between the Kondo and Fano effects. For obvious reasons their most dramatic competition occurs when energy of the side-coupled quantum dot coincides with the Kondo resonance, i.e. for $\varepsilon_{2}=0$. Quantum interference effects destroy then the Kondo peak washing out the zero-bias enhancement of the Andreev conductance.
We have studied a unique nature in which the Fanotype quantum interference manifest itself in the energy spectrum and differential conductance of the heterojunction where a metallic lead is coupled via double quantum dot to superconducting electrode. In the regime of subgap source-drain voltage $|e V|<|\Delta|$ nonequilibrium charge transport is contributed only through the anomalous Andreev channel when electron from the metallic electrode is converted into the Cooper pair (propagating further in superconductor) with a simultaneous reflection of hole back to the metallic lead. Transmittance of such Andreev scattering is a sensitive probe of the proximity induced on-dot pairing as well as the quantum interference and correlation effects.

Since on-dot pairing mixes the particle with hole states the interference effects are doubled in a comparison to similar junctions without the superconducting electrode. In particular, for $T$-shape configuration schematically shown in figure 1 we notice that effective spectrum of the interfacial quantum dot develops the resonance and antiresonance, correspondingly at $\pm \varepsilon_{2}$ (figure 21). These Fano-type structures are present whenever the hopping integral $t$ to the side-attached quantum $\operatorname{dot}(i=2)$ is much smaller than the linebroadening $\Gamma_{N}$ (whereas $\Gamma_{S}$ merely controls the induced quasiparticle splitting). Upon increasing $t$ the Fano-type features disappear, evolving into the new quasiparticle peaks (figure (3) being a consequence of the proximity effect indirectly spread onto the side-attached quantum dot.

Correlation effects play an important role with regard to the following aspects: a) the charging effect which causes appearance of the Coulomb satellite near $\varepsilon_{1}+U_{1}$, b) the Kondo singlet state (when the interfacial quantum dot spin is effectively screened by electrons of the metallic electrode leading to formation of a narrow resonance at $\left.\mu_{N}\right)$, and c) eventual suppression the on-dot pairing. We have previously shown [16] that the Kondo effect enhances the zero-bias Andreev conductance as indeed reported experimentally [5]. In the present work we indicate that in the double quantum dots the quantum interference can (destructively) affect such feature if the Fano-type structures appear nearby the Kondo peak.

A more detailed analysis of the Fano-Kondo interplay could be a challenging task in the future studies. For this purpose one should resort either to nonperturbative techniques, like the numerical renormalization group, or to some sophisticated perturbative methods capable to interpolate between the limits $t \rightarrow 0, \Gamma_{\beta} \rightarrow 0$ and $U \rightarrow 0$.

\section{Acknowledgments}

We thank B. Bułka and K.I. Wysokiński for instructive discussions on the Fano resonances in nanophysics. This work is partly supported by the Polish Ministry of Science and Education under the grant NN202187833. 


\section{Appendix: Selfenergy of the noncorrelated DQD}

Using the standard Nambu notation we can express the retarded Green's functions of the metallic lead

$$
g_{N}^{r}(\mathbf{k}, \omega)=\left(\begin{array}{cc}
\frac{1}{\omega-\xi_{\mathbf{k} N}} & 0 \\
0 & \frac{1}{\omega+\xi_{\mathbf{k} N}}
\end{array}\right)
$$

the (unperturbed) side-attached quantum dot

$$
g_{2}^{r}(\omega)=\left(\begin{array}{cc}
\frac{1}{\omega-\varepsilon_{2}} & 0 \\
0 & \frac{1}{\omega+\varepsilon_{2}}
\end{array}\right)
$$

and the isotropic superconductor

$$
g_{S}^{r}(\mathbf{k}, \omega)=\left(\begin{array}{cc}
\frac{u_{\mathbf{k}}^{2}}{\omega-E_{\mathbf{k}}}+\frac{v_{\mathbf{k}}^{2}}{\omega+E_{\mathbf{k}}} & \frac{-u_{\mathbf{k}} v_{\mathbf{k}}}{\omega-E_{\mathbf{k}}}+\frac{u_{\mathbf{k}} v_{\mathbf{k}}}{\omega+E_{\mathbf{k}}} \\
\frac{-u_{\mathbf{k}} v_{\mathbf{k}}}{\omega-E_{\mathbf{k}}}+\frac{u_{\mathbf{k}} v_{\mathbf{k}}}{\omega+E_{\mathbf{k}}} & \frac{u_{\mathbf{k}}^{2}}{\omega+E_{\mathbf{k}}}+\frac{v_{\mathbf{k}}^{2}}{\omega-E_{\mathbf{k}}}
\end{array}\right)
$$

In the equation $\mathrm{A.3}$ we applied the BCS coefficients

$$
\begin{aligned}
u_{\mathbf{k}}^{2}, v_{\mathbf{k}}^{2} & =\frac{1}{2}\left[1 \pm \frac{\xi_{\mathbf{k} S}}{E_{\mathbf{k}}}\right] \\
u_{\mathbf{k}} v_{\mathbf{k}} & =\frac{\Delta}{2 E_{\mathbf{k}}},
\end{aligned}
$$

where $E_{\mathbf{k}}=\sqrt{\xi_{\mathbf{k} S}^{2}+\Delta^{2}}$.

For the case of uncorrelated quantum dots $\left(U_{i}=0\right)$ we can determine the selfenergy $\Sigma^{0}(\omega)$ of the interfacial quantum dot from the following equation

$$
\boldsymbol{\Sigma}^{0}(\omega)=\sum_{\mathbf{k}, \beta=N, S} V_{\mathbf{k}, \beta} g_{\beta}^{r}(\mathbf{k}, \omega) V_{\mathbf{k}, \beta}^{*}+t g_{2}^{r}(\omega) t^{*} .
$$

Assuming the wide-band limit we introduce the constant weighed density of states

$$
2 \pi \sum\left|V_{\mathbf{k} \beta}\right|^{2} \delta\left(\omega-\xi_{\mathbf{k}, \beta}\right)=\left\{\begin{array}{l}
\Gamma_{\beta} \text { for }\left|\xi_{\mathbf{k}, \beta}\right|<D / 2 \\
0 \text { elsewhere }
\end{array}\right.
$$

where $D$ is the conduction bandwidth. We then easily find that

$$
\sum_{\mathbf{k}} V_{\mathbf{k}, N} g_{N}^{r}(\mathbf{k}, \omega) V_{\mathbf{k}, N}^{*}=\left(\begin{array}{cc}
\frac{-i \Gamma_{N}}{2} & 0 \\
0 & \frac{-i \Gamma_{N}}{2}
\end{array}\right)
$$

because, according to the Kramers-Krönig relation, the real part disappears. In the same way we obtain from a straightforward algebra that [2]

$$
\sum_{\mathbf{k}} V_{\mathbf{k}, S} g_{S}^{r}(\mathbf{k}, \omega) V_{\mathbf{k}, S}^{*}=\frac{\gamma(\omega) \Gamma_{S}}{2 i}\left(\begin{array}{cc}
-1 & \frac{\Delta}{\omega} \\
\frac{\Delta}{\omega} & -1
\end{array}\right)
$$

where

$$
\gamma(\omega)=\frac{|\omega| \Theta(|\omega|-\Delta)}{\sqrt{\omega^{2}-\Delta^{2}}}-\frac{i \omega \Theta(\Delta-|\omega|)}{\sqrt{\Delta^{2}-\omega^{2}}} .
$$

In the extreme subgap limit $|\omega| \ll \Delta$ the function (A.8) approaches $\gamma(\omega) \rightarrow-i \omega / \Delta$ and in consequence

$$
\lim _{|\omega| \ll \Delta} \Sigma^{0}(\omega)=\left(\begin{array}{cc}
\frac{-i \Gamma_{N}}{2}+\frac{|t|^{2}}{\omega-\varepsilon_{2}} & \frac{-\Gamma_{S}}{2} \\
\frac{-\Gamma_{S}}{2} & \frac{-i \Gamma_{N}}{2}+\frac{|t|^{2}}{\omega+\varepsilon_{2}}
\end{array}\right)
$$

which proves the equation (6).
[1] M. Pustilnik and L.I. Glazman, J. Phys.: Condens. Matter 16, R513 (2004).

[2] Y. Yamada, Y. Tanaka, and N. Kawakami, arXiv/1101.5239 (2011).

[3] P.W. Anderson, P.A. Lee, M. Randeria, T.M. Rice, N. Trivedi, F.C. Zhang, J. Phys.: Condens. Matter 16, R755 (2004).

[4] Y.V. Nazarov, Y.L. Blanter, Quantum Transport: Introduction to Nanosciene, Cambridge University Press, Cambridge (2009).

[5] R.S. Deacon, Y. Tanaka, A. Oiwa, R. Sakano, K. Yoshida, K. Shibata, K. Hirakawa, and S. Tarucha, Phys. Rev. Lett. 104, 076805 (2010); R.S. Deacon, Y. Tanaka, A. Oiwa, R. Sakano, K. Yoshida, K. Shibata, K. Hirakawa, and S. Tarucha, Phys. Rev. B 81, 121308(R) (2010) and the supplemented on-line information.

[6] R. Fazio and R. Raimondi, Phys. Rev. Lett. 80, 2913 (1998); Phys. Rev. Lett. 82, 4950 (1999); P. Schwab and R. Raimondi, Phys. Rev. B 59, 1637 (1999).

[7] A.A. Clerk, V. Ambegaokar, and S. Hershfield, Phys. Rev. B 61, 3555 (2000).

[8] J.C. Cuevas, A. Levy Yeyati, and A. Martin-Rodero, Phys. Rev. B 63, 094515 (2001).

[9] M. Krawiec and K.I. Wysokiński, Supercond. Sci. Technol. 17, 103 (2004).
[10] Y. Tanaka, N. Kawakami, and A. Oguri, J. Phys. Soc. Jpn. 76, 074701 (2007).

[11] J. Bauer, A. Oguri, and A.C. Hewson, J. Phys.: Condens. Matter 19, 486211 (2007).

[12] T. Hecht, A. Weichselbaum, J. von Delft, and R. Bulla, J. Phys. Condens. Matter 20, 275213 (2008).

[13] Q.-F. Sun, J. Wang, and T.-H. Lin, Phys. Rev. B 59, 3831 (1999); Q.-F. Sun, H. Guo, and T.-H. Lin, Phys. Rev. Lett. 87, 176601 (2001).

[14] S.Y. Cho, K. Kang, and C.-M. Ryu, Phys. Rev. B 60, 16874 (1999).

[15] Y. Avishai, A. Golub, and A.D. Zaikin, Phys. Rev. B 63, 134515 (2001); T. Aono, A. Golub, and Y. Avishai, Phys. Rev. B 68, 045312 (2003).

[16] T. Domański and A. Donabidowicz Phys. Rev. B 78, 073105 (2008); T. Domański, A. Donabidowicz, and K.I. Wysokiński, Phys. Rev. B 78, 144515 (2008); Phys. Rev. B 76, 104514 (2007).

[17] V. Koerting, B.M. Andersen, K. Flensberg, and J. Paaske, Phys. Rev. B 82, 245108 (2010).

[18] M.R. Graeber, T. Nussbaumer, W. Belzig, and C. Schönenberger, Nanotechnology 15, 479 (2004).

[19] L. Hofstetter, A. Geresdi, M. Aagesen J. Nygard, C. Schönenberger, and S. Csonka, Phys. Rev. Lett. 104, 246804 (2010). 
[20] L.G. Herrmann, F. Portier, P. Roche, A. Levy Yeyati, T. Kontos, and C. Strunk, Phys. Rev. Lett. 104, 026801 (2010).

[21] J. Eldridge, M.G. Pala, M. Governale, J. König, Phys. Rev. B 82, 184507 (2010).

[22] B. Sothmann, D. Futterer, M. Governale, J. König, Phys. Rev. B 82, 094514 (2010).

[23] Y. Yamada, Y. Tanaka, and N. Kawakami, J. Phys. Soc. Jpn. 79, 043705 (2010).

[24] A.E. Miroshnichenko, S. Flach, and Y.S. Kivshar, Rev. Mod. Phys. 82, 2257 (2010).

[25] Y. Tanaka, N. Kawakami, and A. Oguri, Phys. Rev. B 78, 035444 (2008); J. Phys.: Conf. Series 150, 022086 (2009).

[26] Y. Tanaka, N. Kawakami, and A. Oguri, Phys. Rev. B
81, 075404 (2010).

[27] B. Bułka, P. Stefański, Phys. Rev. Lett. 86, 5128 (2001).

[28] R. Žitko, Phys. Rev. B 81, 115316 (2010).

[29] P. Trocha and J. Barnaś, Phys. Rev. B 76, 165432 (2007).

[30] T. Meng, S. Florens, and P. Simon, Phys. Rev. B 79, 224521 (2009).

[31] H. Haug and A.-P. Jauho, Quantum Kinetics in Transport and Optics of Semiconductors, Springer Verlag, Berlin (1996).

[32] S. Sasaki, H. Tamura, T. Akazaki, and T. Fujisawa, Phys. Rev. Lett. 103, 266806 (2009).

[33] A. Karmanyos, I. Grace, and C.J. Lambert, Phys. Rev. B 79, 075119 (2009). 


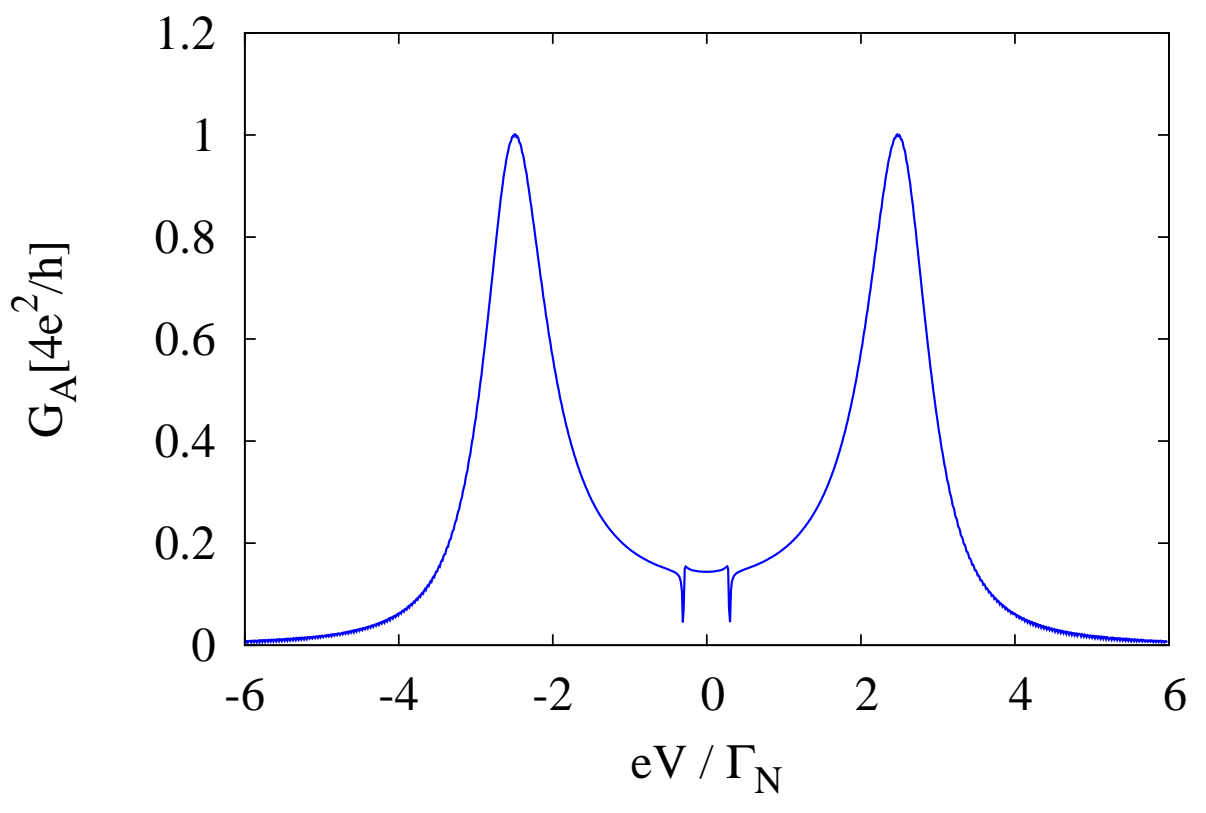

